\title{
Inclusion Body Myositis Masquerading as Amyotrophic Lateral Sclerosis
}

\author{
Kerri L. Schellenberg, Wendy S.W. Johnston, Sanjay Kalra, Lothar Resch, Edward S. Johnson
}

Can. J. Neurol. Sci. 2010; 37: 687-691

Amyotrophic lateral sclerosis (ALS) is a devastating diagnosis with a relentless progression toward death. Inclusion body myositis (IBM) is often cited as a common mimicker of $\mathrm{ALS}^{1}$, although literature to support this is scant. Despite extensive diagnostic evaluation, without a muscle biopsy, misdiagnoses may still occur. As we will discuss, patients with IBM may have hyperreflexia and fasciculations which are often mistaken for signs of motor neuron disease. The distinctive pattern of weakness in IBM, particularly the weakness of finger flexion, may be initially lacking. Lastly, spontaneous activity and large, long duration motor units may be seen on electromyography (EMG). These findings may be interpreted as "neuropathic" and contribute to diagnostic error. ${ }^{2}$ We report two patients who were initially diagnosed with ALS, but on reevaluation at the ALS clinic, were confirmed to have IBM.

\section{Case 1}

A 76-year-old man was referred to a multidisciplinary ALS clinic in the fall of 2006 for progressive weakness. He required a walker for ambulating long distances, and had difficulty rising from the floor and climbing stairs. He experienced weakness in his hands, and was unable to lift heavy objects. He denied cramps. He had sensory symptoms involving only his right fifth digit. There were no bulbar symptoms, no dyspnea, and his weight was stable. Bowel and bladder function was intact.

Past medical history was significant for 60 years of diabetes mellitus type 1 and probable diabetic polyneuropathy. He had suffered a stroke with mild residual dysarthria and mild right hemiparesis. He also had hypertension, hypothyroidism, hyperlipidemia, gastroesophageal reflux disease, a familial tremor, and ataxia from gentamicin induced ototoxicity. His family history was unremarkable for neurological disease. There were no clear toxic exposures.

On examination, the cranial nerves were intact aside from mild dysarthria. Bulk was reduced diffusely, particularly in right greater than left ulnar innervated intrinsic muscles and the quadriceps. The tone was normal in all four limbs. Strength at the neck and shoulder was intact, but power at the elbows and wrists was 4/5 according to Medical Research Council grading. Finger extensors and flexors were symmetric at 4-/5. First dorsal interossei were $3 / 5$. Hip flexion and ankle dorsiflexion were $4 / 5$, but knee flexion and extension were intact. Fasciculations were restricted to the first dorsal interossei. He had a right greater than left postural tremor that increased with finger-nose testing, and also had a mild head tremor. Foot tapping was mildly slow bilaterally. In the arms, reflexes were diminished at bilateral brachioradialis, normal at the right biceps and left triceps, and increased at the right triceps. Knee jerks were diminished and ankle jerks were absent. Plantar responses were flexor bilaterally, and Hoffman's and palmomental signs were negative. He had decreased pinprick and light touch sensation over bilateral fifth digits, decreased vibratory sensation to the toes, and had a stocking distribution of diminished pinprick and light touch sensation to the mid calf. Coordination was essentially normal. Stance and gait were wide based.

Investigations included elevated creatine kinase and erythrocyte sedimentation rate levels at 1183 (reference range $<200 \mathrm{U} / \mathrm{L})$ and $31(0-20 \mathrm{~mm} / \mathrm{h})$ respectively. The serum angiotensin converting enzyme was also increased at 49 (10-40 $\mathrm{U} / \mathrm{L})$. The serum protein electrophoresis was normal. He had normal calcium and creatinine levels. His rheumatoid factor was minimally increased at $21(<20 \mathrm{kU} / \mathrm{L})$, but the antinuclear antibodies, antineutrophil cytoplasmic antibody, anti-Jo-1 and anti-smooth muscle levels were normal. His thyroid stimulating hormone was elevated at $6.25(0.2-4.0 \mathrm{mU} / \mathrm{L})$, but the free thyroxine 4 was normal. Serum vitamin $B_{12}$ levels, and urine mercury, arsenic and lead levels were normal. His fasting glucose and hemoglobin A1c were increased at 8.8 (3.3-6.0 $\mathrm{mmol} / \mathrm{L})$ and $7.5(4.3-6.1 \%)$ respectively. His forced vital capacity was significantly reduced at $35 \%$ of expected. The computed tomography of his chest showed chronic changes presumed due to a granulomatous infection. The magnetic resonance imaging of the brain, cervical and lumbar spine did not reveal an alternate diagnosis.

Nerve conduction studies revealed a sensorimotor axonal polyneuropathy, and bilateral compressive ulnar neuropathies at the elbows. Electromyography (EMG) showed spontaneous activity in the form of fibrillations and positive sharp waves which were graded as follows; multiple right upper extremity $(1+$ to $3+)$, left thoracic paraspinal $(1+)$, and bilateral lower extremity muscles $(1+$ to $4+)$. No spontaneous activity was found in the left arm or masseter. On the first EMG the motor units were thought to be "neurogenic" with rare motor units demonstrating slightly increased amplitude and reduced

From the Department of Medicine, Division of Neurology (KLS, WSWJ, SK), Department of Laboratory Medicine and Pathology (LR, ESJ), University of Alberta, Edmonton, Alberta, Canada.

Received November 24, 2009. Final Revisions Submitted March 22, 2010. Correspondence to: Kerri L. Schellenberg, Division of Neurology, University of Alberta, 2E3 WMC, 8440 - 112 St., Edmonton, Alberta, T6G 2B7, Canada. 


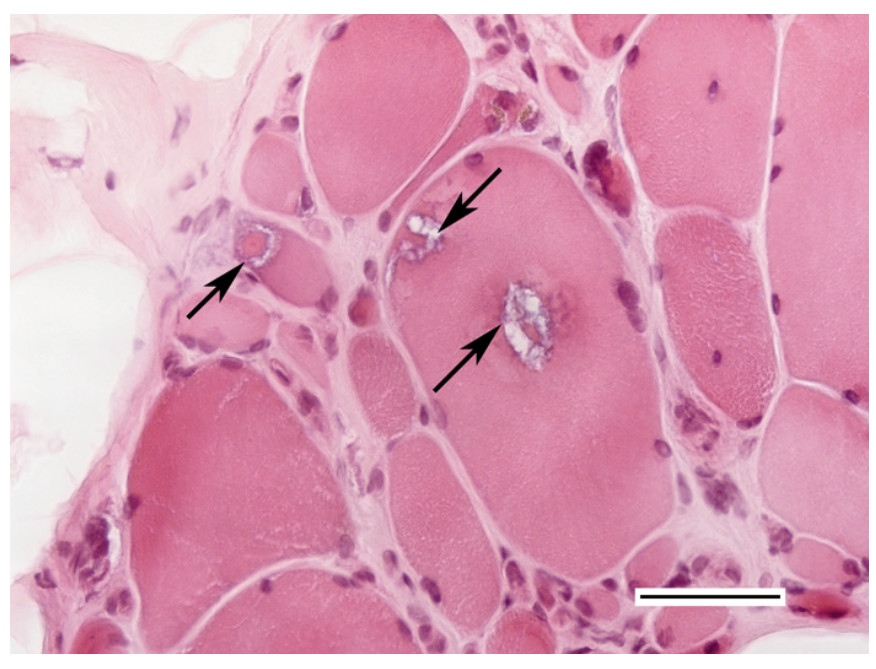

Figure 1: Photomicrograph of $H \& E$ stained cryostat section showing myofiber size variation, endomysial fibrosis and typical inclusion bodies (inclusion bodies indicated by arrows). Calibration bar equals $50 \mu$. recruitment, and polyphasia was noted in many motor units. On a second EMG study performed four months later in a different laboratory by a different physician, fibrillations, positive sharp waves, and occasional motor units of small amplitude, early recruitment and polyphasia were seen. This prompted an investigation into myopathic causes, and a muscle biopsy was obtained which was consistent with IBM. Light microscopical examination revealed myopathic changes with fatty infiltration of the perimysium, patchy endomysial fibrosis, myofiber hypertrophy and atrophy and increased internal nuclei. Focal endomysial mononuclear cell infiltration was seen with occasional invasion of intact myofibers by CD8(+) lymphocytes. Many myofibers revealed granular basophilic intracytoplasmic material often at the periphery of a clear vacuole; this material was red on the modified muscle trichrome stain (Figures 1 and 2). Electromicroscopy revealed this material to correspond to cyto-membranous bodies, and cytoplasmic filamentous structures measuring 15-20nm typical of inclusion body were observed.

Despite the initial diagnosis of ALS, the pathological and clinical diagnosis was IBM. The patient died from an acute coronary syndrome and renal failure. A post mortem evaluation was not obtained.

\section{Case 2}

A 51-year-old man was seen in a multidisciplinary ALS clinic in March 2005. He had several months' history of progressive weakness including difficulty climbing stairs, rising from a kneeling position and lifting weights with his arms. He described exertional dyspnea without symptoms of nocturnal hypoventilation. He experienced dysphagia, but had no dysarthria or sialorrhea. He denied muscle twitching or cramps, but had longstanding generalized aches. Bowel and bladder function was unaffected.

His past medical history was remarkable for celiac disease. There was no family history of ALS.

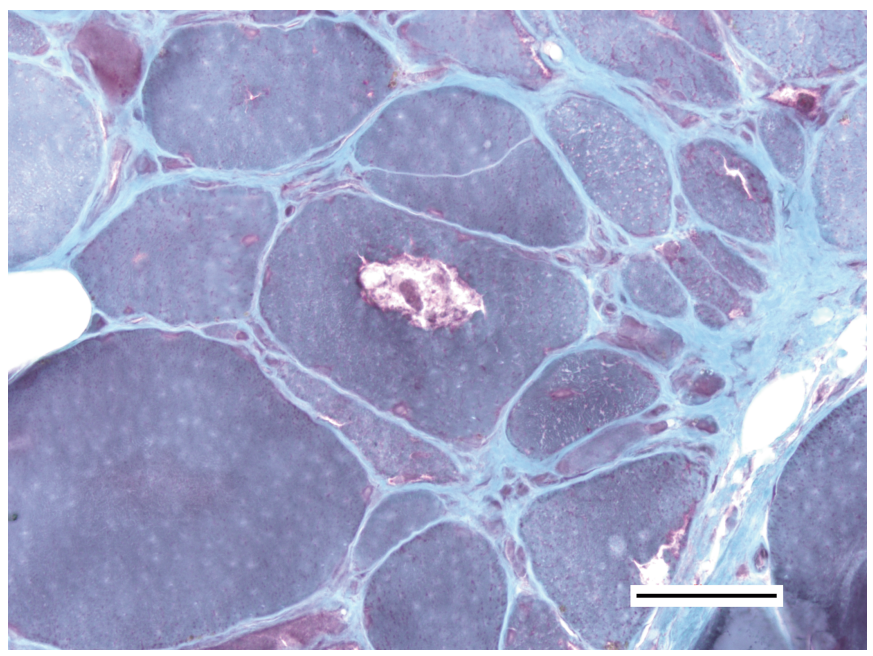

Figure 2: Photomicrograph of modified muscle trichrome stain of cryostat section showing myofiber size variation, endomysial fibrosis and large rimmed vacuole in myofiber (center of image). Calibration bar equals $50 \mu$.

On examination, the cranial nerves were normal. Bulk and tone were normal in all four extremities. Fasciculations were not observed. Power was reduced symmetrically for movements at the shoulder, elbow and wrist at grades between 4 - to $4+/ 5$. The first dorsal interrosei were strong, and the finger flexor strength was not initially recorded. Hip flexion strength was normal and knee flexion and extension were $4 / 5$. The distal leg muscles were strong. Deep tendon reflexes were normal at the biceps and ankle, and increased at the triceps, brachioradialis and knees. The left Hoffman's was more prominent than the right. The plantar responses were flexor. Sensation was normal for light touch and temperature, but vibration and proprioception was mildly reduced at the toes. Tandem walking was slightly unsteady.

On follow-up, the finger flexor strength was 4+ on the right and 4- on the left. Leg strength had declined to a hip flexion of $2 / 5$ and knee flexion and extension of $3 / 5$ with quadriceps atrophy. The tibialis anterior muscle strength was $4 / 5$. Fasciculations were now noted in the right arm.

Investigations included a normal complete blood count, electrolytes, creatinine, thyroid stimulating hormone and vitamin $\mathrm{B}_{12}$. The rheumatoid factor was elevated at $299(<20 \mathrm{kU} / \mathrm{L})$ with a positive antinuclear antibody, but the complement levels were normal. Testing for human immunodeficiency virus, syphillis, Tay Sachs disease, and urinary heavy metals was negative. Pulmonary function testing revealed a forced vital capacity of $92 \%$ of predicted, and an extensive cardiac and pulmonary work up failed to determine an etiology for his exertional dyspnea. The computerized tomography of the chest showed a small density of uncertain significance. The echocardiogram did not reveal a significant abnormality. Magnetic resonance imaging of the cervical spine showed spondylosis at levels $5 / 6$ without cord or root impingement.

Nerve conduction studies were normal. The initial EMG was thought to be neurogenic based upon increased insertional 
activity, fibrillations, positive sharp waves, and polyphasia in all muscles tested in the right arm and leg, as well as fibrillations, positive sharp waves, and complex repetitive discharges in the left thoracic paraspinals. Additionally, several muscles demonstrated increased motor unit potential amplitude, while reduced recruitment was observed in the right iliopsoas. However, a subsequent EMG performed in a different laboratory one year later revealed early recruitment and decreased motor unit amplitude at the left biceps and vastus medialis, with polyphasia seen in the vastus medialis. Early recruitment and polyphasia was also observed in the left tibialis anterior. Fibrillations and positive sharp waves were seen in all three muscles (graded as $1+$ to $3+$ ). Myotonic discharges were observed in the left tibialis anterior and complex repetitive discharges and early recruitment were seen in the left vastus medialis. A muscle biopsy was obtained due to the evolving clinical picture (progressive finger flexor and knee extensor weakness with quadriceps atrophy) as well as the findings on repeat EMG which were suggestive of a myopathic etiology. The biopsy was consistent with the diagnosis of IBM. The muscle biopsy showed myofiber atrophy and hypertrophy with mainly small, rounded, randomly dispersed myofibers but with occasional small angulated myofibers. This was accompanied by endomysial fibrosis and multi-focal dense mononuclear inflammatory cell infiltrates comprised primarily of CD8(+) lymphocytes which also occasionally invaded intact myofibers (Figure 3). Vacuoles rimmed with basophilic granular material (red with muscle trichrome staining) were readily found. Electron microscopical examination revealed cyto-membranous inclusions and intracytoplasmic filaments typical of IBM (Figure 4).

Although this man was initially diagnosed with ALS, the slow progression of symptoms, the pattern of muscle weakness and the second EMG findings suggested an alternate diagnosis of IBM that was confirmed pathologically.

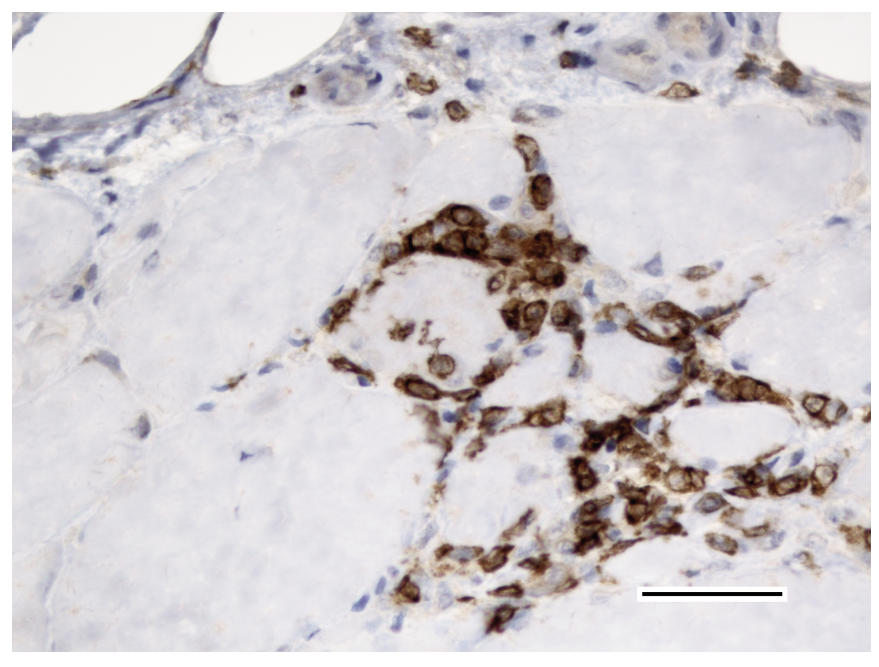

Figure 3: Photomicrograph of CD8 immunolabeled cryostat section showing endomysial lymphocytic infiltrate and infiltration of nonnecrotic myofiber, center of image, by CD8 positive cells. Calibration bar equals $50 \mu$.

\section{Review of the Literature}

A literature search of the Medline and Embase databases was performed using the terms amyotrophic lateral sclerosis, inclusion body myositis, differential diagnosis and diagnostic errors. Web of Science was also searched in order to find further publications that cited these articles. When review articles were excluded, only three articles with 13 cases listed in total were discovered.

Dabby et al retrospectively reviewed the charts of 70 patients with a pathological diagnosis of inclusion body myositis. ${ }^{3}$ Nine $(13 \%)$ of these patients had originally been diagnosed as having motor neuron disease. Eight of these nine patients had finger flexor weakness, but in only one patient was this weakness profound at onset. If weakness began in the legs, then proximal groups including the quadriceps were affected. Six had asymmetrical weakness. None had bulbar symptoms at the onset of disease. Four patients eventually developed dysphagia, but none was dysarthric. Fasciculations were observed in two patients, and none had muscle cramps. Preserved reflexes in weak limbs were seen in six patients, hyperactive reflexes in two patients, but the authors state none had definite upper motor neuron signs. The creatine kinase was mildly to moderately elevated in four patients. Spontaneous activity was seen in the EMG of all patients, and long duration motor units were seen in eight. Although a myopathic cause was not suspected on the basis of the routine EMG, quantitative EMG revealed myopathic units in four of the five patients tested. Muscle biopsies were performed on the basis of slow progression or prominent finger flexor weakness.

Ludolph and Knirsch describe one case report of a patient who was found to have IBM after being initially diagnosed as having ALS. ${ }^{4}$ He developed asymmetrical distal arm weakness and atrophy which spread proximally and eventually affected all limbs with distal greater than proximal involvement. Bilateral hand flexors were graded at $4 / 5$. Mild distal vibration loss was

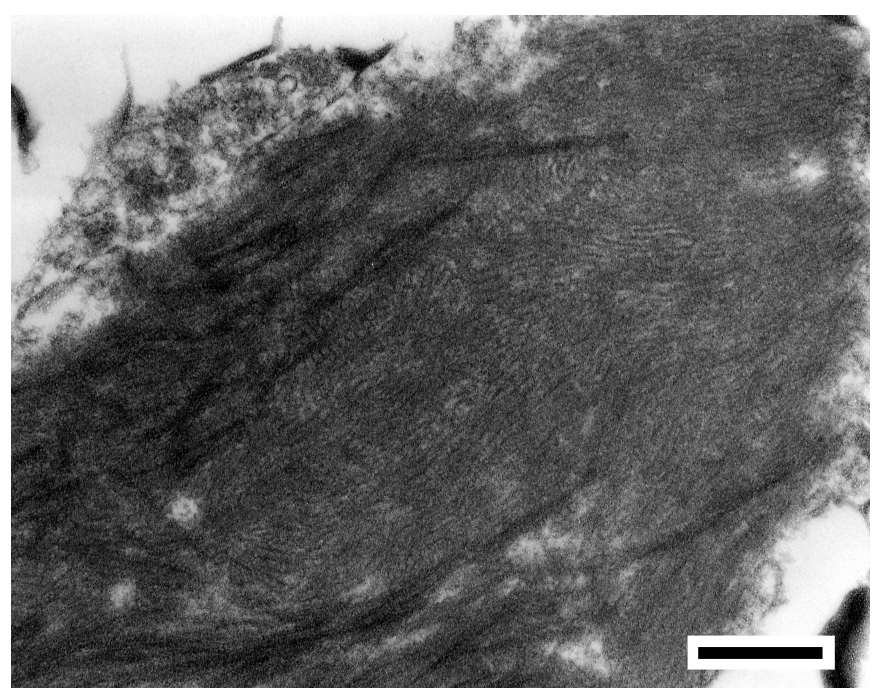

Figure 4: Electron micrograph showing typical tubulo-filamentous cytoplasmic inclusion with tubulo-filaments measuring 17-20nm. Calibration bar equals 320nm. 
also found. The creatine kinase was normal, and the nerve conduction studies were unremarkable. The EMG was "neurogenic" with a few positive sharp waves and fibrillation potentials. A muscle biopsy was obtained due to the slow disease progression, the lack of bulbar symptoms, upper motor neuron signs, or hand flexor involvement, and absence of florid denervation on EMG. The biopsy was consistent with IBM.

Cafforio et al observed the clinical, laboratory and histopathological association of hereditary inclusion body myopathy and motor neuron disease in three patients. ${ }^{5}$ All had muscle weakness, atrophy and hyperreflexia for at least one reflex tested. All patients had fasciculations noted either clinically or on EMG. One had dysarthria, dysphagia, cognitive impairment and urge incontinence. This patient was also reported to have a spastic gait and hyperreflexia. Aside from the hyperreflexia, no other upper motor neuron signs were reported for the other two cases. Reduced motor unit amplitude, a study "compatible with a neuronopathy pattern", and absent sural sensory responses were found on nerve conduction studies. Spontaneous activity was noted on the EMG studies of all three cases. The first case underwent motor evoked potentials that revealed pyramidal tract involvement, and arm somatosensoryevoked potentials showed cervical reduced amplitudes. Another patient had reduced amplitude of cortical responses with somatosensory-evoked potentials. All biopsies revealed the accumulation of phosphorylated neurofilaments and rimmed vacuoles without inflammatory infiltrate. Angulated myofibers and group atrophy were noted. The authors speculated that the presence of both neurogenic atrophy and pathological findings consistent with IBM pointed to concurrent neuronal and muscular degeneration suggesting a common pathological mechanism for these cases.

\section{DiscuSSION}

Inclusion body myositis is the most common myopathy in patients over 50 years-of-age. Males are more frequently affected than females. ${ }^{6}$ Weakness and atrophy preferentially affect the quadriceps, wrist and finger flexors, ankle dorsiflexors, and are commonly asymmetrical. Up to $40 \%$ of patients may complain of dysphagia. A concomitant sensory neuropathy is seen in up to $30 \%$ of patients. ${ }^{7}$ Associated autoimmune disorders occur in up to $32 \%$ of patients, and some patients have been found to have paraproteinemias and immunodeficiency. ${ }^{8}$ There does not appear to be an increased incidence of malignancy. The life expectancy of patients with IBM is relatively normal. ${ }^{7}$ There is no proven therapy for IBM. ${ }^{9}$ The creatine kinase may be elevated, but usually does not rise above ten times the upper limit of normal. ${ }^{7}$ The EMG demonstrates increased spontaneous activity, brief small polyphasic units or large polyphasic units. The nerve conduction studies may have low compound motor action potential amplitudes or may also show decreased conduction velocities. ${ }^{6}$ The muscle biopsy shows an inflammatory cell infiltrate comprised primarily of CD8(+) Tcells inflammatory infiltrates, ragged red fibers, rimmed vacuoles, and amyloid and ubiquitin deposits. On electron microscopy, characteristic $17-20 \mathrm{~mm}$ tubulofilaments are seen in the cytoplasm or nuclei. ${ }^{10}$

Despite the fact that IBM is often cited as a common mimicker of motor neuron disease, there is a relative paucity of case reports substantiating this in the literature. Our literature review revealed only three articles with 13 cases listed in total. We speculate on two possible explanations for this disparity. It may be thought too common to be worthwhile reporting, or perhaps it is not quite as common as previously believed.

Regardless, several interesting observations can be made. Aside from the cases presented by Cafforio et al, ${ }^{5}$ which describe the possible association of two diseases, all patients including ours were initially diagnosed as ALS and later found to have IBM. ${ }^{3,4}$ The reason for obtaining a muscle biopsy in most cases was clinical suspicion based upon the pattern of weakness, particularly of hand and finger flexors, and slow progression of disease. Also cited was the lack of bulbar or definite upper motor neuron involvement. Additionally, we suspected a muscular etiology based on EMG findings of small motor units and early recruitment, while others have noted the lack of florid denervation and an abnormal quantitative EMG (despite a normal routine EMG) as suspicious for IBM.

Despite the diagnosis of IBM, we observed hyperreflexia in both of our cases. Although the unilateral hyperreflexia observed in the first case was likely secondary to a previous stroke, no alternative etiology was found to explain our second patient's hyperreflexia. Dabby et al noted hyperactive reflexes or preserved reflexes in weak limbs in all but one case. ${ }^{3}$ Ludolph and Knirsch did not comment on their patient's reflexes, ${ }^{4}$ but at least one reflex was brisk in all cases presented by Cafforio et al. ${ }^{5}$ This is a useful observation as many sources indicate that reflexes in IBM are diminished. The presence of hyperreflexia does not necessarily exclude the diagnosis of IBM, although alternative explanations must be excluded. It is important to continually evaluate patients' strength and progression of disease so as not to overlook IBM as an alternative diagnosis. The pattern of weakness may evolve over time as evidenced by our cases. It must also be noted that only one out of nine cases presented by Dabby et al had profound finger flexor weakness on initial examination. ${ }^{3}$ Additionally, although dysphagia was reported in several patients, dysarthria was noted only in our patient who had residual deficits from a stroke, and in one case presented by Cafforio et al. ${ }^{5}$ It appears that the presence of dysarthria in IBM, if possible, is exceedingly rare, and alternate diagnoses should be explored. Although fasciculations are commonly associated with ALS, the presence of fasciculations does not exclude IBM as a possible diagnosis. In our first case, fasciculations were restricted to ulnar nerve innervated muscles in the presence of ulnar neuropathies, but no alternative etiology was uncovered to explain the fasciculations seen in our second case. Fasciculations were also noted in two cases reported by Dabby et al, and all patients presented by Cafforio et al.,5 Additionally, routine muscle biopsy in the diagnostic evaluation of suspected ALS is not recommended provided discerning clinical follow-up and judicious repeated EMG studies are undertaken.

We have presented two patients who were initially diagnosed with ALS, but later found to have IBM on both clinical and pathological grounds. We performed a literature search investigating the occurrence of other patients with IBM first misdiagnosed as ALS. Although IBM is often cited as a common mimicker of motor neuron disease, there is a paucity of case reports substantiating this allegation in the literature. We have 
discussed several clinical implications based on our study of the current literature. Namely, IBM should remain in the differential diagnosis even with the presence of hyperreflexia, fasciculations, and an EMG that superficially appears to be "neurogenic". Additionally, the distinctive pattern of weakness in IBM, particularly weakness of finger flexion, may be initially lacking thus contributing to the diagnostic error. Slow progression of disease, and an emerging pattern of weakness consistent with IBM in the absence of evolving upper motor neuron signs should alert the clinician to the possibility of IBM in a patient carrying the diagnosis of ALS. Repeat EMG may serve to clarify the diagnosis. To avoid misdiagnosis, it is important to be mindful of the variability in clinical presentation of IBM.

\section{ACKNOWLEDGEMENTS}

Supported by the ALS Society of Canada (Dr. K. Schellenberg - Betty Norman Fellowship recipient).

\section{REFERENCES}

1. Chad DA. Classification, diagnosis, and presentation of diagnosis. In: Mitsumoto $\mathrm{H}$, Przedborski S, Gordon PH, editors. Amyotrophic Lateral Sclerosis. New York: Tayor \& Francis Group; 2006. 1st ed. p. 207-15.

2. Needham M, Mastaglia FL. Inclusion body myositis: current pathogenetic concepts and diagnostic and therapeutic approaches. Lancet Neurol. 2007; 6:620-31.

3. Dabby R, Lange DJ, Trojaborg W, Hays AP, Lovelace RE, Brannagan $\mathrm{TH}$, et al. Inclusion body myositis mimicking motor neuron disease. Arch Neurol. 2001; 58:1253-6.

4. Ludolph AC, Knirsch U. Problems and pitfalls in the diagnosis of ALS. J Neurol Sci. 1999; 165:S14-20.

5. Cafforio G, Pistolesi S, D'Avino C, Galluzzi F, Patricelli A, Solito B, et al. Inclusion body myopathy associated with motor neuron syndrome: three case reports. Clin Neuropathol. 2005; 24:36-41.

6. Engel WK, Askanas V. Inclusion-body myositis: clinical, diagnostic, and pathologic aspects. Neurology. 2006; 66:S20-9.

7. Amato AA, Greenberg SA. Inflammatory myopathies. In: Miller $\mathrm{AE}$, editor. Muscle diseases. Continuum: lifelong learning in neurology. Philadelphia: Lippincott Williams \& Wilkins; 2006. Vol 12: p. 140-68.

8. Dalakas MC. Inflammatory, immune, and viral aspects of inclusionbody myositis. Neurology. 2006; 66:S33-8.

9. Griggs RC. The current status of treatment for inclusion-body myositis. Neurology. 2006; 66:S30-2.

10. Lidov H, De Girolami U, Gherardi R. Skeletal muscle diseases. In: Gray F, De Girolami U, Poirier J, editors. Escourolle and Poirier manual of basic neuropathology. Philadelphia: Elsevier; 2004. 4th ed. p. 309-11. 